\title{
A General Factor of Brain White Matter Integrity Predicts Information Processing Speed in Healthy Older People
}

\author{
Lars Penke, ${ }^{1,2,3}$ Susana Muñoz Maniega, ${ }^{2,3,4}$ Catherine Murray, ${ }^{1}$ Alan J. Gow, ${ }^{1,2}$ Maria C. Valdés Hernández, ${ }^{2,3,4}$ \\ Jonathan D. Clayden, ${ }^{5}$ John M. Starr, ${ }^{2,6}$ Joanna M. Wardlaw, ${ }^{2,3,4}$ Mark E. Bastin, ${ }^{2,3,4,7}$ and Ian J. Deary ${ }^{1,2,3}$ \\ ${ }^{1}$ Department of Psychology and ${ }^{2}$ Centre for Cognitive Ageing and Cognitive Epidemiology, The University of Edinburgh, Edinburgh EH8 9JZ, United \\ Kingdom, ${ }^{3}$ Scottish Imaging Network, A Platform for Scientific Excellence (SINAPSE) Collaboration, ${ }^{4}$ SFC Brain Imaging Research Centre, Department of \\ Clinical Neurosciences, The University of Edinburgh, Edinburgh EH4 2XU, United Kingdom, ${ }^{5}$ Institute of Child Health, University College London WC1N \\ 1EH, London, United Kingdom, ${ }^{6}$ Geriatric Medicine Unit, The University of Edinburgh, Edinburgh EH4 2DN, United Kingdom, and ${ }^{7}$ Department of \\ Medical and Radiological Sciences (Medical Physics), The University of Edinburgh, Edinburgh EH4 2XU, United Kingdom
}

Human white matter integrity has been related to information processing speed, but it is unknown whether impaired integrity results from localized processes or is a general property shared across white matter tracts. Based on diffusion MRI scans of 132 healthy individuals with a narrow age range around 72 years, the integrity of eight major white matter tracts was quantified using probabilistic neighborhood tractography. Principal component analyses (PCAs) were conducted on the correlations between the eight tracts, separately for four tract-averaged integrity parameters: fractional anisotropy, mean diffusivity, and radial and axial diffusivity. For all four parameters, the PCAs revealed a single general factor explaining $\sim 45 \%$ of the individual differences across all eight tracts. Individuals' scores on a general factor that captures the common variance in white matter integrity had significant associations with a general factor of information processing speed for fractional anisotropy $(r=-0.24, p=0.007)$ and radial diffusivity $(r=0.21, p=0.016)$, but not with general intelligence or memory factors. Individual tracts showed no associations beyond what the common integrity factor explained. Just as different types of cognitive ability tests share much of their variance, these novel findings show that a substantial amount of variance in white matter integrity is shared between different tracts. Therefore, impaired cortical connection is substantially a global process affecting various major tracts simultaneously. Further studies should investigate whether these findings relate more to the role of tract integrity and information processing speed in nonpathological cognitive aging or in lifelong-stable processes.

\section{Introduction}

Fast and efficient information processing between different brain areas is a prerequisite of higher cognitive abilities. White matter pathways connecting brain networks provide a foundation for such abilities (Jung and Haier, 2007; Deary et al., 2010). The integrity of individual brain white matter pathways can be quantified using probabilistic tractography based on diffusion MRI

\footnotetext{
Received March 26, 2010; revised April 14, 2010; accepted April 21, 2010.

L.P., S.M.M., C.M., and M.V.H. were funded by the United Kingdom Medical Research Council. L.P., A.J.G., and the LBC1936 data collection were supported by The Disconnected Mind (www.disconnectedmind.ed.ac.uk) funded by Help the Aged and Research into Ageing. The imaging was performed in the SFC Brain Imaging Research Centre (www.sbirc.ed.ac.uk) supported by the Scottish Funding Council as a center in the SINAPSE (Scottish Imaging Network, A Platform for Scientific Excellence, www.sinapse.ac.uk) Collaboration. J.M.W. is partially funded by the Scottish Funding Council as part of the SINAPSE Collaboration. This work has made use of the resources provided by the Edinburgh Compute and Data Facility (www.ecdf.ed.ac.uk), which is partially supported by the edikt initiative (www.edikt.org.uk). The work was undertaken within The University of Edinburgh Centre for Cognitive Ageing and Cognitive Epidemiology, part of the cross-council Lifelong Health and Wellbeing Initiative. Funding from the Biotechnology and Biological Sciences Research Council, Engineering and Physical Sciences Research Council, Economic and Social Research Council, and Medical Research Council is gratefully acknowledged. We thank the study secretary Paula Davies; Janie Corley, Caroline Brett, Alison Pattie, and Ross Henderson for data collection and data entry; the nurses and other staff at the Wellcome Trust Clinical Research Facility, the radiographers and other staff of the SFC Brain Imaging Research Centre; and the staff at Lothian Health Board and at the SCRE Centre, University of Glasgow.

Correspondence should be addressed to Dr. Lars Penke, Centre for Cognitive Ageing and Cognitive Epidemiology, Department of Psychology, The University of Edinburgh, 7 George Square, Edinburgh EH8 9JZ, United Kingdom. E-mail: lars.penke@ed.ac.uk.

DOI:10.1523/JNEUROSCI.1553-10.2010

Copyright $\odot 2010$ the authors $\quad 0270-6474 / 10 / 307569-06 \$ 15.00 / 0$
}

(Behrens et al., 2007; Jones, 2008). Also in line with distributed brain networks, information processing speed, as assessed by simple reaction time or inspection time tasks, is robustly associated with intelligence (Jensen, 2006). There is some evidence that processing speed is associated with measures of white matter integrity (for review, see Madden et al., 2009a). However, it is not clear from the existing studies whether lowered white matter integrity results from localized microstructural processes that affect single tracts in some individuals, or whether it is to some extent a global phenomenon that affects many tracts simultaneously, as a recent report of positive correlations between tract integrity parameters measured across a range of association and projection pathways suggests (Wahl et al., 2010). Furthermore, it is unclear whether specific white matter tracts or the integrity that might be shared across tracts are especially important for higher cognitive functioning and speed of information processing.

An indirect indication that integrity shared across major white matter networks might underlie higher cognitive ability comes from the psychometric literature. There, it has been shown repeatedly that ability tests across all cognitive domains share $\sim 50 \%$ of their variance between people, allowing the extraction of a general cognitive ability factor from almost any correlation matrix of cognitive tests (Carroll, 1993; Jensen, 1998). The existence of a strong general factor at the level of cognitive differences makes it plausible that more general, brain-wide individual dif- 
ferences, like shared white matter integrity differences across tracts, underlie some of the differences in higher cognitive functioning.

White matter integrity and speed of information processing are especially prone to aging effects, and thus it has been proposed that age-related cognitive decline may occur as a result of cortical disconnection (O'Sullivan et al., 2001; Madden et al., 2009a). Age-related decline in speed of information processing has been identified as an important variable that predates general age-related cognitive decline (Salthouse and Ferrer-Caja, 2003; Finkel et al., 2007) and is probably less affected by compensatory neurocognitive processes (Park and Reuter-Lorenz, 2009).

Here, we hypothesize that different white matter tracts share a substantial amount of integrity across the aging brain, i.e., older individuals who have high integrity in one tract will tend to have higher integrity in all others. Furthermore, we hypothesize that this shared variance in white matter tract integrity across tracts, if it exists, should be associated with faster information processing speed.

\section{Materials and Methods}

Subjects. The participants were 132 generally healthy, older individuals ( 68 men, 64 women, mean age $=71.8$ years, $\mathrm{SD}=0.4$, range $71.0-72.8)$ from the Lothian Birth Cohort 1936 (Deary et al., 2007). All were right handed, Caucasian, living independently in the community, and without signs of dementia or mild cognitive impairment, based on self-reports and Mini-Mental State Examination scores above 23 (Folstein et al., 1975). Written informed consent was obtained from all participants under protocols approved by the National Health Service ethics committees (MREC and LREC).

Neuroimaging. Subjects underwent diffusion MRI on a GE Signa HDX 1.5T MRI clinical scanner using a self-shielding gradient set with maximum gradient strength of $33 \mathrm{mT} \cdot \mathrm{m}^{-1}$, and eight-channel head array coil. Echo-planar diffusion-weighted images $\left(b=1000 \mathrm{~s} \cdot \mathrm{mm}^{-2}\right)$ were acquired in 64 noncollinear directions, along with seven T2-weighted images $\left(b=0 \mathrm{~s} \cdot \mathrm{mm}^{-2}\right)$. Seventy-two contiguous axial 2-mm-thick slices were acquired at $2 \times 2 \times 2 \mathrm{~mm}$ resolution. Repetition time was $16.5 \mathrm{~s}$ and echo time $95.5 \mathrm{~ms}$.

Datasets were preprocessed using FSL tools (FMRIB; http://www. fmrib.ox.ac.uk/) to extract the brain, remove bulk subject motion- and eddy current-induced artifacts, and estimate water diffusion tensor parameters (Basser et al., 1994). The BEDPOST/ProbTrack tractography algorithm (Behrens et al., 2007) with a two-fiber model and 5000 streamlines was used to reconstruct tracts of interest. An automatic tract selection method with good reproducibility (Clayden et al., 2009a), based on a model of tract topology (Clayden et al., 2007; Bastin et al., 2008), was used to generate equivalent tracts of interest in each subject. This technique, termed probabilistic neighborhood tractography, optimizes the choice of seed point for tractography by estimating the best matching tract from a series of candidates against a reference tract derived from a digital human white matter atlas (Hua et al., 2008), as described by Muñoz Maniega et al. (2008). The topological tract model was also used to reject false-positive connections (Clayden et al., 2009b). Eight white matter pathways in the brain thought to be related to cognitive functioning were segmented: genu and splenium of the corpus callosum and frontal white matter connections bilaterally (cingulum bundles, uncinate fasciculus, and arcuate fasciculus, with the latter mostly covering the so-called "Geschwind area"). Using the eigenvalues $(\lambda 1, \lambda 2, \lambda 3)$ of the apparent water diffusion tensor, tract-averaged values of fractional anisotropy $(\mathrm{FA})$, mean diffusivity $(\langle D\rangle)$, and the axial $(\lambda \mathrm{ax}=\lambda 1)$ and radial $(\lambda \mathrm{rad}=\{\lambda 2+\lambda 3\} / 2)$ diffusivities were calculated for all eight tracts in each subject. The component diffusivities, $\lambda$ ax and $\lambda \mathrm{rad}$, represent water diffusion parallel and perpendicular to the axonal fibers and may help differentiate impaired myelination from axonal injury (Bastin et al., 2009; Madden et al., 2009a), although care should be taken interpreting them in terms of underlying tissue structure (WheelerKingshott and Cercignani, 2009).

Cognitive testing. Information processing speed was assessed with three well established tasks. Reaction times were assessed using a standalone device (Deary et al., 2001). Simple reaction time (averaged across 20 trials) required pressing a button as fast as possible when a " 0 " was displayed on an LCD screen. Four-choice reaction time (averaged across correct responses in 40 trials) required pressing the correct button out of four as fast as possible when a number from 1 to 4 was displayed on an LCD screen. Inspection time (correct responses across 150 trials, stimulus exposure time $=6-200 \mathrm{~ms}$ ) is a two-alternative, forced-choice, backward-masked visual discrimination task that requires indicating, without response time pressure, which of two parallel, vertical lines of markedly different lengths was longer (Deary et al., 2007). Performance scores on all three tasks were submitted to principal component analysis (PCA), which yielded a strong common factor, explaining $57.2 \%$ of the variance (for factor loadings, see Fig. 1A). Higher values on this factor indicate slower information processing speed.

Intelligence was assessed by six subtests from the Wechsler Adult Intelligence Scale III ${ }^{\mathrm{UK}}$ : Symbol Search, Digit Symbol, Matrix Reasoning, Letter-Number Sequencing, Digit Span Backwards, and Block Design (Wechsler, 1998a). The first unrotated principal component (explained variance $=48.9 \%$ ) was extracted from these six subtests and interpreted as a general intelligence factor (Jensen, 1998).

Finally, memory was assessed by six subtests of the Wechsler Memory Scale III ${ }^{\text {UK }}$ : Logical Memory immediate and delayed recall, Spatial Span forward and backward, and Verbal Paired Associates I (first recall) and II (Wechsler, 1998b). A general memory factor was extracted as the first unrotated principal component (explained variance $=36.82 \%$ ).

\section{Results}

We ran four separate PCAs on all eight white matter tracts, one for each indicator of their integrity (as is customary, the principal components will be referred to as factors throughout). In every case the results indicated a clear one-factor solution (Fig. 1), with 
Table 1. Tract loadings, explained variance, and mean between-tract Pearson correlations for the general white matter integrity factors based on four different water diffusion parameters

\begin{tabular}{lcccc}
\hline & FA & $\langle D\rangle$ & $\lambda \mathrm{ax}$ & $\lambda$ rad \\
\hline Left arcuate fasciculus & 0.71 & 0.77 & 0.73 & 0.76 \\
Right arcuate fasciculus & 0.70 & 0.78 & 0.71 & 0.77 \\
Left cingulum bundle & 0.60 & 0.73 & 0.79 & 0.67 \\
Right cingulum bundle & 0.67 & 0.83 & 0.77 & 0.81 \\
Left uncinate fasciculus & 0.56 & 0.58 & 0.71 & 0.52 \\
Right uncinate fasciculus & 0.73 & 0.70 & 0.74 & 0.69 \\
Genu corpus callosum & 0.63 & 0.69 & 0.77 & 0.64 \\
Splenium corpus callosum & 0.42 & 0.17 & 0.20 & 0.22 \\
Explained variance & $40.28 \%$ & $46.98 \%$ & $49.18 \%$ & $43.38 \%$ \\
Mean between-tract $r$ & 0.31 & 0.36 & 0.39 & 0.33 \\
\hline
\end{tabular}

the first unrotated factor explaining between $40.3 \%$ (FA) and $49.2 \%$ ( $\lambda$ ax) of the variance (Table 1). All eight tracts showed substantial positive loadings on each of these four factors. The splenium of the corpus callosum consistently showed the lowest loadings (Table 1). Factor structures (i.e., the pattern of factor loadings of the eight tracts on the first unrotated principal components) were almost identical for the four different water diffusion parameters (congruence coefficient $\varphi=0.986-0.999$ ) (see also Table 1$)$ and for men and women $(\varphi=0.966-0.991)$. Thus, almost half of the integrity variance is shared between major white matter tracts; there is a general factor of white matter integrity.

After controlling for gender and age in days at testing, the general processing speed factor correlated significantly with the common FA factor $(r=-0.24, p=0.007)$ (Fig. 2) and showed a nonsignificant trend with the common $\langle D\rangle$ factor $(r=0.16, p=$ $0.06)$. The nature of the latter correlation was clarified by the factors based on the directional diffusivity subcomponents: the common $\lambda \mathrm{rad}$ factor correlated significantly with the processing speed factor $(r=0.21, p=0.016)$, whereas the common $\lambda$ ax factor did not $(r=0.05, p=0.55)$, a difference in correlation size that is statistically significant $\left[t_{(129)}=2.214, p\right.$ (two-tailed) $=$ $0.029]$. The three individual tasks that comprise the general processing speed factor showed a very similar pattern of results, though the correlation between inspection time and the common FA factor showed only a trend $(r=0.15, p=0.094)$, and only four-choice reaction time was significantly correlated with the common $\lambda$ rad factor $(r=0.19, p=0.031)$ (Table 2). Neither the general intelligence factor nor the general memory factor were significantly correlated with any of the four tract integrity factors ( $p$ values $>0.30$ ), indicating dissociation of global white matter tract integrity with higher cognitive functions (Table 2).

To explore a posteriori whether the correlations between common white matter tract integrity and processing speed was carried by especially strong associations between speed and the integrity of individual tracts, we calculated all possible 8 (individual tracts) $\times 4$ (white matter integrity parameters) $=32$ correlations of tracts with the general speed factor. Of these, six correlations (with left arcuate and left uncinate fasciculus FA, $\langle D\rangle$, and $\lambda \mathrm{rad}$ ) were statistically significant ( $p$ values $<0.05$ ), but not particularly strong $(r$ values $<0.26)$, and 30 out of 32 were in the expected direction. This pattern further supports the notion that the associations of the common tract integrity factors with processing speed are shared among the tracts we studied.

Furthermore, after statistically controlling for the common tract integrity factor in correlations between the processing speed factor and integrity of the eight individual tracts, only left uncinate fasciculus FA still showed even a statistical trend $(p=0.07)$.
A
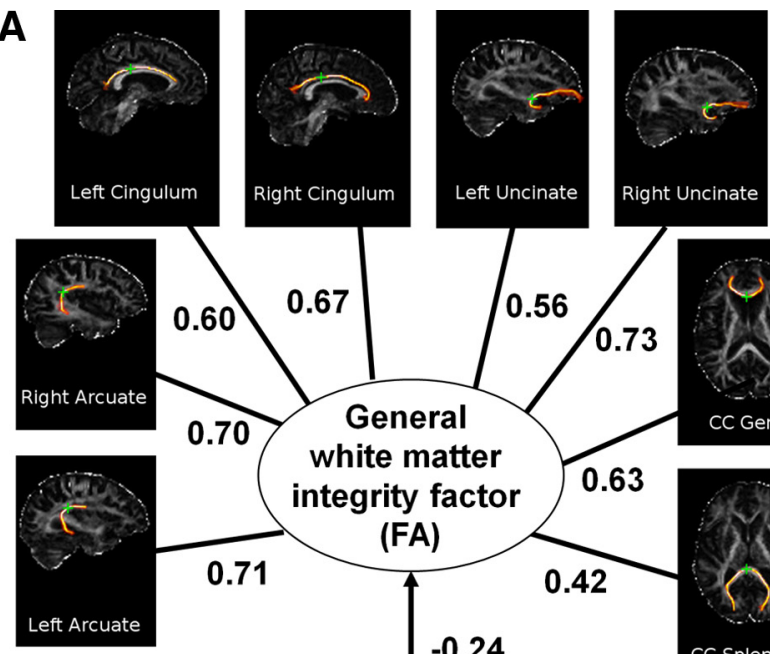

0.67

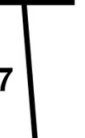

rar

0.71

General

$0.56 / 0.73$

white matter integrity factor (FA)

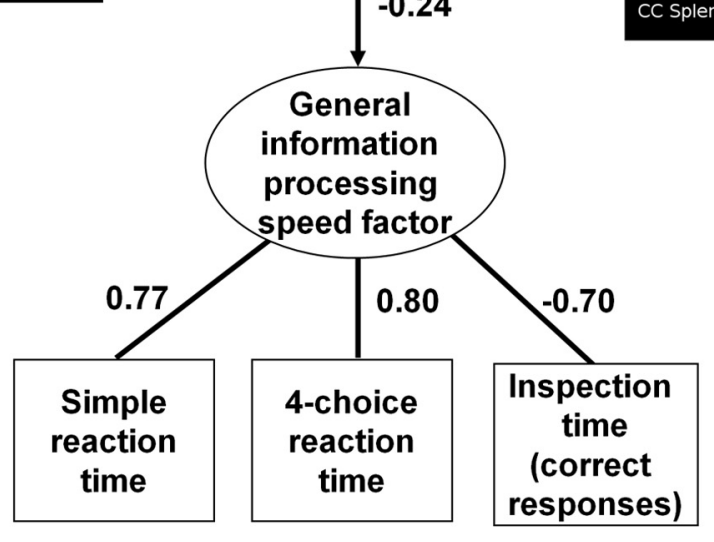

B

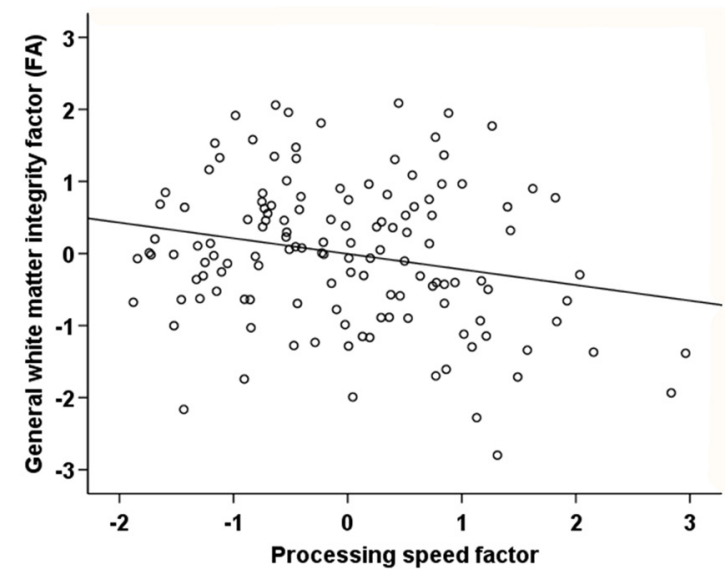

Figure 2. The FA-based white matter integrity factor and its association with information processing speed. $\boldsymbol{A}$, Brain images show white matter tract segmentations obtained in one representative participant. Seed points are marked with a green cross and tracts are projected into the plane of the seed point. The statistics on the lines are the factor loadings of the average $F A$ values of the eight tracts on the latent white matter integrity factor (top) and of the three measures of processing speed on the latent speed factor. The number on the two-headed arrow connecting the two factors is their correlation. $B$, Scatter plot of the relationship between the $F A$-based white matter integrity factor and the processing speed factor.

In addition, previously nonsignificant correlations of the right cingulum bundle with the speed factor became significant $[$ for $\langle D\rangle$ and $\lambda$ ax ( $p$ values $<0.05)$; statistical trend $(p=0.09)$ for $\lambda \mathrm{rad}$ ]. Thus, contributions of the integrity of individual tracts to processing speed were only very limited over and above what was explained by the common tract integrity factors. 


\begin{tabular}{|c|c|c|c|c|}
\hline & $F A$ & $\langle D\rangle$ & $\lambda \mathrm{ax}$ & $\lambda \mathrm{rad}$ \\
\hline General processing speed factor & $\begin{array}{l}-0.24 \\
(-0.40,-0.07)\end{array}$ & $\begin{array}{c}0.16 \\
(-0.01,0.32)\end{array}$ & $\begin{array}{c}0.05 \\
(-0.12,0.22)\end{array}$ & $\begin{array}{c}0.21 \\
(0.04,0.38)\end{array}$ \\
\hline Simple reaction time (log) & $\begin{array}{l}-0.18 \\
(-0.34,-0.01)\end{array}$ & $\begin{array}{c}0.10 \\
(-0.07,0.27)\end{array}$ & $\begin{array}{c}0.00 \\
(-0.17,0.17)\end{array}$ & $\begin{array}{c}0.14 \\
(-0.03,0.31)\end{array}$ \\
\hline Four-choice reaction time & $\begin{array}{l}-0.19 \\
(-0.35,-0.02)\end{array}$ & $\begin{array}{c}0.17 \\
(0.00,0.33)\end{array}$ & $\begin{array}{c}0.10 \\
(-0.07,0.27)\end{array}$ & $\begin{array}{c}0.19 \\
(0.02,0.35)\end{array}$ \\
\hline Inspection time (correct responses) & $\begin{array}{c}0.15 \\
(-0.02,0.31)\end{array}$ & $\begin{array}{l}-0.09 \\
(-0.26,0.08)\end{array}$ & $\begin{array}{l}-0.01 \\
(-0.18,0.16)\end{array}$ & $\begin{array}{l}-0.13 \\
(-0.04,0.29)\end{array}$ \\
\hline General intelligence factor & $\begin{array}{c}0.08 \\
(-0.09,0.25)\end{array}$ & $\begin{array}{l}-0.06 \\
(-0.23,0.11)\end{array}$ & $\begin{array}{l}-0.05 \\
(-0.22,0.12)\end{array}$ & $\begin{array}{l}-0.07 \\
(-0.24,0.10)\end{array}$ \\
\hline General memory factor & $\begin{array}{l}-0.03 \\
(-0.20,0.14)\end{array}$ & $\begin{array}{l}-0.02 \\
(-0.19,0.15)\end{array}$ & $\begin{array}{l}-0.05 \\
(-0.22,0.12)\end{array}$ & $\begin{array}{c}0.00 \\
(-0.17,0.17)\end{array}$ \\
\hline
\end{tabular}

Values in parentheses are $95 \%$ confidence intervals of the correlations. Lower values on the general processing speed factor and in simple and four-choice reaction time as well as higher values in inspection time indicate better information processing speed.

While we defined our speed factor exclusively by chronometric cognitive tasks, Symbol Search and Digit Symbol are often also regarded as (psychometric) speed tasks. Indeed these two tests loaded on both factors approximately equally. Even though neither Symbol Search nor Digit Symbol correlated significantly with the four tract integrity factors ( $p$ values $>0.09$ ), moving both tests from the general intelligence factor to the speed factor did not have any noteworthy effects on the results reported here.

Finally, we tested whether any of these effects were gender specific. With regard to the cognitive variables reported in Table 2 , men and women differed only, and weakly, in their mean performance on the inspection time task (men: mean $=115.74$, $\mathrm{SD}=9.32$; women: mean $=112.20, \mathrm{SD}=10.77 ; t_{(131)}=2.03$, $p=0.045)$. There were no significant gender differences in the white matter integrity factor scores ( $p$ values $>0.05$ ). We reran all analyses without controlling for gender and tested interaction effects with gender instead. None of these were statistically significant ( $p$ values $>0.05$ ), indicating that all reported relationships were similar for men and women.

\section{Discussion}

It is well established that, among nonclinical populations, people who perform poorly in one cognitive domain tend to perform poorly in others too, which allows for the extraction of a general factor of cognitive ability differences (Carroll, 1993; Jensen, 1998). Here, for the first time, we show that the same is true for white matter integrity: low integrity in every tract we studied was positively correlated with lower integrity in all other tracts, which allowed the extraction of a general factor for each of the four water diffusion parameters we measured. While this does not mean that all tracts across the brain will show exactly the same degree of white matter integrity, thereby permitting some regional differences as in the well established age-related anteriorposterior gradient (Sullivan and Pfefferbaum, 2006), these results do suggest that individual differences in white matter integrity are to a substantial degree common to different tracts, not just a phenomenon that primarily affects specific individual tracts. Furthermore, while it has been shown in some studies that information processing speed relates to white matter integrity of the whole brain (Vernooij et al., 2009), broad regions of interest (Deary et al., 2006), and individual tracts (Correia et al., 2008; Turken et al., 2008), our study adds the novel result that it is shared integrity across tracts that is associated with speed rather than the integrity of specific tracts. Global tract integrity was exclusively associated with processing speed, but not with higher cognitive abilities like general intelligence and memory, perhaps because speed tends to be affected earlier in life by age-related decline (Salthouse and Ferrer-Caja, 2003; Finkel et al., 2007), whereas higher abilities are more likely to be maintained by compensatory processes (Park and Reuter-Lorenz, 2009). Further studies will be needed to understand this dissociation.

Correlations of white matter integrity with information processing speed were stronger with FA than with $\langle D\rangle$, which suggests that mild microstructural alterations of white matter, such as minor fiber loss without gross tissue loss, are responsible for these associations. Also, correlations were limited to shared radial, but not shared axial diffusivity, which would be consistent with interstitial water increase or possibly early demyelination rather than neuronal loss or axonal damage, as an early feature in this disconnection process (Burzynska et al., 2010).

White matter integrity was especially shared among tracts with frontal projections, like the arcuate fasciculus and cingulum bundle, but less with more posterior white matter fibers like the splenium of the corpus callosum (Table 1). This is in line with the prominent role of the prefrontal cortex in higher cognitive functions (Gray and Thompson, 2004) and the parietofrontal integration theory of intelligence (Jung and Haier, 2007; Deary et al., 2010). However, this does not imply that posterior white matter tracts play no role in higher cognitive functions. Luders et al. (2007), for example, found that the integrity of posterior, but not anterior, regions of the corpus callosum were associated with intelligence. Also, an earlier analysis of the same sample has found that a single nucleotide polymorphism in the adrenergic receptor gene $A D R B 2$ was associated specifically with integrity of the splenium of corpus callosum, with a partial mediation effect on age-related decline in intelligence (Penke et al., 2010). These results could be a sign of different, more specific processes affecting the integrity of the splenium of corpus callosum and subsequently higher cognitive functions that do not affect frontal white matter tracts, which could explain why the splenium loaded relatively weakly on the general white matter integrity factors in the current study. Integrity variance of tracts in the left and right hemispheres were shared with the common factors to a similar degree, indicating that general white matter impairments affect both hemispheres alike (cf. Wahl et al., 2010).

Our results were found in an older, generally healthy sample without signs of mild cognitive impairment or dementia. It remains to be investigated whether the results extend to patients with clinical conditions like stroke, dementia, or neurodegenera- 
tive disorders. Our sample had a very narrow age range in the early seventies, which suggests the interpretation that the results we found are related to normal cognitive aging and age-related decline in white matter integrity (Raz and Rodrigue, 2006; Deary et al., 2009; Madden et al., 2009b). However, since our data were cross-sectional and based on a single cohort, we were unable to test aging effects directly. Therefore, a cognitive aging interpretation of our results remains speculative, and similar studies in younger age-homogenous groups will be useful in testing this. The integrity variance shared by major white matter tracts in the present study was associated with processing speed, often found to be a leading indicator of cognitive aging (Salthouse and FerrerCaja, 2003; Finkel et al., 2007). This supports that the general disconnection of brain networks (O'Sullivan et al., 2001), and potentially the deterioration of organism-wide system integrity (Christensen et al., 2001), might be one underlying "common cause" of normal age-related cognitive decline. However, there is also evidence that processing speed is related to cognitive ability at all ages (Jensen, 1998), and thus the general white matter integrity we found in this study could also be a lifelong-stable biological foundations of processing speed, regardless of age. Longitudinal studies or comparisons of different age-homogeneous groups are needed to disentangle these alternative hypotheses.

There is some evidence that age-related differences in FA and $\langle D\rangle$ are driven primarily by increased radial, but not axial, diffusivity (Bhagat and Beaulieu, 2004; Madden et al., 2009a), which matches the pattern of correlations with processing speed found in the current study. That white matter integrity was especially shared among frontally projecting tracts in our elderly sample is in line with anterior-posterior gradient usually found in brain aging (Sullivan and Pfefferbaum, 2006).

A limitation of the current study is that we have not examined other factors potentially coassociated with cognitive impairments and aging, such as white matter lesions, because a larger sample will be required to adjust for these factors. However, in this healthy sample few subjects had many white matter lesions. In a large elderly cohort, Vernooij et al. (2009) showed that more subtle white matter differences detected by diffusion tensor MRI provided incremental value in explaining age-related cognitive decline over the more macrostructural white matter changes in lesions.

The current study showed that nonpathological individual differences in white matter integrity that are related to processing speed - a basic aspect of cognitive functioning - are to a substantial degree shared by different major white matter tracts. That white matter integrity is more of a global, brain-general than tract-specific feature can have major implications for our understanding of normal differences in cognitive ability and cognitive aging. Causal factors that affect white matter tracts, like small vessel disease or hypertension, might to a substantial degree do so in a global, systemic manner, which might in turn help to explain the biological underpinning of the general factor of cognitive ability. However, from the present study it is as yet unclear whether a general factor of white matter integrity can be equally well identified in different clinical samples and different age groups, and whether or not associations with speed generalize across these different groups. It will be interesting to discover in future longitudinal studies and age-heterogeneous crosssectional studies whether the above results are more related to lifelong-stable differences in cognitive ability or cognitive aging.

\section{References}

Basser PJ, Mattiello J, LeBihan D (1994) MR diffusion tensor spectroscopy and imaging. Biophys J 66:259-267.

Bastin ME, Piatkowski JP, Storkey AJ, Brown LJ, Maclullich AM, Clayden JD (2008) Tract shape modelling provides evidence of topological change in corpus callosum genu during normal ageing. Neuroimage 43:20-28.

Bastin ME, Clayden JD, Pattie A, Gerrish IF, Wardlaw JM, Deary IJ (2009) Diffusion tensor and magnetization transfer MRI measurements of periventricular white matter hyperintensities in old age. Neurobiol Aging 30:125-136.

Behrens TEJ, Berg HJ, Jbabdi S, Rushworth MFS, Woolrich MW (2007) Probabilistic diffusion tractography with multiple fibre orientations: what can we gain? Neuroimage 34:144-155.

Bhagat YA, Beaulieu C (2004) Diffusion anisotropy in subcortical white matter and cortical gray matter: changes with aging and the role of CSFsuppression. J Magn Reson Imaging 20:216-227.

Burzynska AZ, Preuschhof C, Bäckman L, Nyberg L, Li SC, Lindenberger U, Heekeren HR (2010) Age-related differences in white matter microstructure: region-specific patterns of diffusivity. Neuroimage 49:21042112.

Carroll JB (1993) Human cognitive abilities. Cambridge, UK: Cambridge UP.

Christensen H, Mackinnon AJ, Korten A, Jorm AF (2001) The common cause hypothesis of cognitive aging. Psychol Aging 16:588-599.

Clayden JD, Storkey AJ, Bastin ME (2007) A probabilistic model-based approach to consistent white matter tract segmentation. IEEE Trans Med Imaging 26:1555-1561.

Clayden JD, Storkey AJ, Muñoz Maniega S, Bastin ME (2009a) Reproducibility of tract segmentation between sessions using an unsupervised modelling-based approach. Neuroimage 45:377-385.

Clayden JD, King MD, Clark CA (2009b) Shape modelling for tract selection. Med Image Comput Comput Assist Interv 12:150-157.

Correia S, Lee SY, Voorn T, Tate DF, Paul RH, Zhang S, Salloway SP, Malloy PF, Laidlaw DH (2008) Quantitative tractography metrics of white matter integrity in diffusion-tensor MRI. Neuroimage 42:568-581.

Deary IJ, Der G, Ford G (2001) Reaction times and intelligence differences: a population-based cohort study. Intelligence 29:389-399.

Deary IJ, Bastin ME, Pattie A, Clayden JD, Whalley LJ, Starr JM, Wardlaw JM (2006) White matter integrity and cognition in childhood and old age. Neurology 66:505-512.

Deary IJ, Gow AJ, Taylor MD, Corley J, Brett C, Wilson V, Campbell H, Whalley LJ, Visscher PM, Porteous DJ, Starr JM (2007) The Lothian Birth Cohort 1936: a study to examine influences on cognitive ageing from age 11 to age 70 and beyond. BMC Geriatr 7:28.

Deary IJ, Corley J, Gow AJ, Harris SE, Houlihan LM, Marioni RE, Penke L, Rafnsson SB, Starr JM (2009) Age-associated cognitive decline. Br Med Bull 92:135-152.

Deary IJ, Penke L, Johnson W (2010) The neuroscience of human intelligence differences. Nat Rev Neurosci 11:201-211.

Finkel D, Reynolds CA, McArdle JJ, Pedersen NL (2007) Age changes in processing speed as a leading indicator of cognitive aging. Psychol Aging 22:558-568.

Folstein MF, Folstein SE, McHugh PR (1975) Mini-mental state. A practical method for grading the cognitive state of patients for the clinician. J Psychiatr Res 12:189-198.

Gray JR, Thompson PM (2004) Neurobiology of intelligence: science and ethics. Nat Rev Neurosci 5:471-482.

Hua K, Zhang J, Wakana S, Jiang H, Li X, Reich DS, Calabresi PA, Pekar JJ, van Zijl PC, Mori S (2008) Tract probability maps in stereotaxic spaces: analyses of white matter anatomy and tract-specific quantification. Neuroimage 39:336-347.

Jensen AR (1998) The g factor: the science of mental ability. Westport: Praeger.

Jensen AR (2006) Clocking the mind: mental chronometry and individual differences. Amsterdam: Elsevier.

Jones DK (2008) Studying connections in the living human brain with diffusion MRI. Cortex 44:936-952.

Jung RE, Haier RJ (2007) The parieto-frontal integration theory (P-FIT) of intelligence: converging neuroimaging evidence. Behav Brain Sci 30:135-154.

Luders E, Narr KL, Bilder RM, Thompson PM, Szeszko PR, Hamilton L, Toga AW (2007) Positive correlations between corpus callosum thickness and intelligence. Neuroimage 37:1457-1464. 
Madden DJ, Bennett IJ, Song AW (2009a) Cerebral white matter integrity and cognitive aging: contributions from diffusion tensor imaging. Neuropsychol Rev 19:415-435.

Madden DJ, Spaniol J, Costello MC, Bucur B, White LE, Cabeza R, Davis SW, Dennis NA, Provenzale JM, Huettel SA (2009b) Cerebral white matter integrity mediates adult age differences in cognitive performance. J Cogn Neurosci 21:289-302.

Muñoz Maniega S, Bastin ME, McIntosh A, Lawrie S, Clayden JD (2008) Atlas-based reference tracts improve automatic white matter segmentation with neighbourhood tractography. In: Proceedings of the ISMRM 16th Scientific Meeting and Exhibition, p 3318. Berkeley, CA: ISMRM.

O’Sullivan M, Jones DK, Summers PE, Morris RG, Williams SCR, Markus HS (2001) Evidence for cortical "disconnection" as a mechanism of agerelated cognitive decline. Neurology 57:632-638.

Park DC, Reuter-Lorenz P (2009) The adaptive brain: aging and neurocognitive scaffolding. Annu Rev Psychol 60:173-196.

Penke L, Muñoz Maniega S, Houlihan LM, Murray C, Gow AJ, Clayden JD, Bastin ME, Wardlaw JM, Deary IJ (2010) White matter integrity in the splenium of the corpus callosum is related to successful cognitive aging and partly mediates the protective effect of an ancestral polymorphism in ADRB2. Behav Genet 40:146-156.

Raz N, Rodrigue KM (2006) Differential aging of the brain: patterns, cognitive correlates and modifiers. Neurosci Biobehav Rev 30:730-748.
Salthouse TA, Ferrer-Caja E (2003) What needs to be explained to account for age-related effects on multiple cognitive variables? Psychol Aging 18:91-110.

Sullivan EV, Pfefferbaum A (2006) Diffusion tensor imaging and aging. Neurosci Biobehav Rev 30:749-761.

Turken A, Whitfield-Gabrieli S, Bammer R, Baldo JV, Dronkers NF, Gabrieli JD (2008) Cognitive processing speed and the structure of white matter pathways: convergent evidence from normal variation and lesion studies. Neuroimage 42:1032-1044.

Vernooij MW, Ikram MA, Vrooman HA, Wielopolski PA, Krestin GP, Hofman A, Niessen WJ, Van der Lugt A, Breteler MM (2009) White matter microstructural integrity and cognitive function in a general elderly population. Arch Gen Psychiatry 66:545-553.

Wahl M, Li YO, Ng J, Lahue SC, Cooper SR, Sherr EH, Mukherjee P (2010) Microstructural correlations of white matter tracts in the human brain. Neuroimage 51:531-541.

Wechsler D (1998a) WAIS-III ${ }^{\mathrm{UK}}$ administration and scoring manual. London: Psychological Corporation.

Wechsler D (1998b) WMS-III ${ }^{\text {UK }}$ administration and scoring manual. London: Psychological Corporation.

Wheeler-Kingshott CA, Cercignani M (2009) About "axial" and "radial" diffusivities. Magn Reson Med 61:1255-1260. 